\section{JTI}

JOURNAL OF

TRAUMA AND INJURY

\title{
Comparison of the Surgical Approaches for Frontal Traumatic Intracerebral Hemorrhage
}

\author{
Eun Sung Park, M.D., Seong Keun Moon, M.D., Ph.D., Ki Seong Eom, M.D., Ph.D. \\ Department of Neurosurgery, Wonkwang University Hospital, Iksan, Korea
}

Received: January 18, 2019

Revised: March 18, 2019

Accepted: April 8, 2019

\section{Correspondence to}

Ki Seong Eom, M.D., Ph.D.

Department of Neurosurgery, Wonkwang University Hospital, 895 Muwang-ro, Iksan

54538 , Korea

Tel: $+82-63-859-1467$

Fax: +82-63-852-2606

E-mail:kseom@wonkwang.ac.kr

Purpose: Recent developments in minimally invasive techniques have the potential to reduce surgical morbidity, promote patient recovery, accelerate surgical procedures, and thus improve cost-effectiveness in case management. In this study, we compared the treatment efficacy and results of supraorbital keyhole approach (SOKA) with those of conventional unilateral frontal craniotomy (CUFC) for traumatic intracerebral hemorrhage (TICH) in the frontal lobe.

Methods: We analyzed the data of 38 patients who underwent CUFC $(n=30)$ and SOKA $(\mathrm{n}=8)$ and retrospectively reviewed their medical records and radiological findings. Furthermore, we tried to identify the best surgical method for such lesions by including patients who underwent burr hole aspiration and drainage (BHAD) $(n=9)$ under local anesthesia due to various circumstances.

Results: The difference in the initial Glasgow coma scale score, operative time, and length of hospitalization between the CUFC and SOKA were statistically significant. All radiological features between the two groups including associated skull fracture, amount of pre- and postoperative hematoma, percentage of complete hematoma removal, pre- and postoperative midline shifting of the hematoma, and development of postoperative delayed hematoma were not statistically significant. Our experience of 46 patients with TICH in the frontal lobe with any of the three different surgical methods including BHAD enabled us to obtain valuable findings.

Conclusions: Although it is difficult to insist that one particular approach is more useful than the other, we are confident that SOKA will have more advantages over CUFC in carefully selected patients with frontal TICH depending on the surgical experience of a neurosurgeon.

Keywords: Minimally invasive techniques; Traumatic intracerebral hemorrhage; Supraorbital keyhole approach; Unilateral frontal craniotomy; Burr hole aspiration 


\section{INTRODUCTION}

Traumatic intracerebral hemorrhage (TICH) or contusion occurs in up to $15 \%$ of the patients after traumatic brain injury (TBI) [1]. TICH commonly occurs in the frontal lobe following trauma; however, studies focusing on the effects of TICH in the frontal lobe is limited, except for some case reports $[2,3]$. Although there is no consensus on the various surgical approaches for TICH in the frontal lobe, we believe that frontal craniotomy through bicoronal incision is most likely to be performed. Conventional frontal craniotomy or craniectomy through bicoronal incision can effectively evacuate the hematoma and reduce intracranial pressure (ICP) in patients with severe brain swelling. However, these approaches have disadvantages, such as a longer operative time, more tissue damage, and increased intraoperative blood loss $[3,4]$. Advances in neuroimaging techniques such as microscopy, neuroendoscopy, and neuronavigation have enabled the performance of minimal invasive surgery. Recent developments in minimally invasive techniques have the potential to reduce surgical morbidity, promote patient recovery, accelerate surgical procedures, and thus improve cost-effectiveness in case management [3-5]. In this study, we compared the treatment efficacy and results of supraorbital keyhole approach (SOKA) with those of conventional unilateral frontal craniotomy (CUFC) for TICH in the frontal lobe. Furthermore, we tried to identify the best surgical method for such lesions by including patients who underwent burr hole aspiration and drainage (BHAD) under local anesthesia due to various circumstances.

\section{METHODS}

\section{Data collection and exclusion criteria}

From February 2011 to September 2017, 59 patients underwent operation for unilateral or unilateral dominant bilateral TICH in the frontal lobe at our institute. Of these, we excluded 13 patients who underwent decompressive craniectomy because they often had various concomitant extracranial or brain stem injuries, severe brain injury other than those in the frontal lobe, and had poor neurological condition. Moreover, we excluded eight patients who underwent BHAD because the patients' family did not consent to the operation under general anesthesia due to their circumstances, such as old age, poor health, financial situation, and various other difficult situations. In addition, this BHAD group is considered in the discussion section. We analyzed the data of 38 patients who underwent CUFC $(n=30)$ and SOKA $(n=8)$ and retrospectively reviewed their medical records and radiological findings. We also discussed indications, advantages, and disadvantages for each surgical approach including $\operatorname{BHAD}(n=9)$.

\section{Surgical approach}

\section{CUFC and evacuation of hematoma}

The patients were placed in a supine position, with body slightly upright and the head in $15-20^{\circ}$ retroflexion. Then, a bicoronal bow-like skin incision was made. The skin flap was then retracted anteriorly-inferiorly, and this flap was dissected in the subgaleal plane until the proximal ends of the superciliary arches were reached. At this instance, the pericranium can be raised as a separate, anteriorly pedicled vascularized flap for reconstructive purposes. One burr hole was positioned in the lateral part behind the supraorbital rim. The two additional burr holes positioned at least $1 \mathrm{~cm}$ away from the midline can be used to avoid injury to the superior sagittal sinus. The dura mater was suspended and incised in a curved fashion. The base of the curved incision was oriented towards the eyeball. The contused brain tissue and any hematomas were visualized under a microscope and carefully removed. Strict hemostasis was performed in the surgical field. A drainage tube was inserted after surgery (Fig. 1).

\section{SOKA and evacuation of hematoma}

The patient's head was placed supine on a horseshoe headrest. Then, depending on the location of the lesion, the patient's head is turned $10-25^{\circ}$ to the contralateral side to achieve a straight surgical trajectory and bent $10-15^{\circ}$ posteriorly to achieve the gravity-related self-retraction of the frontal lobe from the skull base. Subsequently, approximately $4 \mathrm{~cm}$ skin incision was started lateral to the supraorbital foramen within the eyebrow, extending 

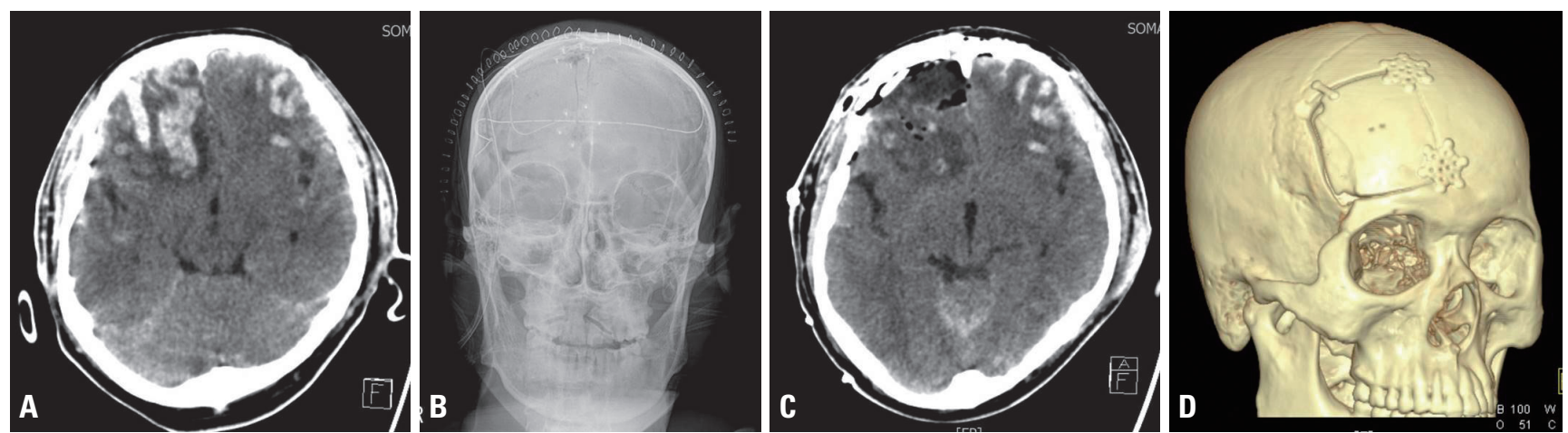

Fig. 1. A 67-year-old man with drowsy state after a fall underwent conventional unilateral frontal craniotomy. (A) Preoperative cranial CT. (B) Postoperative anteroposterior cranial radiograph. (C) Postoperative cranial CT. (D) Cranial three-dimensional reconstruction CT. CT: computed tomography.

laterally to the frontal process of the zygomatic bone following the orbital rim. The temporalis muscle was dissected restrictedly along the superior temporal line to expose parts of the frontal process of the zygomatic bone. By using a high-speed drill, a single fronto-basal burr hole was made lateral to the temporal line. Small frontotemporal craniotomy was made parallel to the orbital rim. After removal of the bone flap, the inner edge of the craniotomy is drilled above the orbital rim, increasing intracranial manipulation. The dura is opened in a curved shape and retracted in a basal direction. Hematomas were evacuated carefully under a microscope. In some cases, a neuroendoscope was used to remove hematomas located in areas not visible under a microscope. After gross total removal of hematoma and contused brain, a gelatin-thrombin matrix sealant $\left(\mathrm{FloSeal}^{\oplus}\right.$; Baxter Inc, Deerfield, IL, USA) was used to facilitate the complete hemostasis. The dura mater was sutured roughly and covered using a suturable dural onlay graft (Lyoplant ${ }^{\circledR}$; Braun, Aesculap, Tutsingen, Germany) (Fig. 2).

\section{Clinical data}

Thirty-eight patients were categorized into two groups (i.e., CUFC and SOKA), and the following demographic and clinical characteristics obtained from the hospital records were compared and analyzed: age, gender, concomitant extracranial injury, cause of injury, Glasgow coma scale (GCS) score at admission and discharge, operative time, length of hospitalization, and mortality. Moreover, we investigated the radiological differentiations based on brain computed tomography (CT) between the two groups, including associated skull fracture, pre- and postoperative day 1 amount of TICH, complete removal of TICH, pre- and postoperative day 1 midline shifting, and postoperative delayed hematoma. The TICH volume was calculated based on a non-enhanced CT image using the formula $\mathrm{A} \times \mathrm{B} \times \mathrm{C} / 2$, where $\mathrm{A}, \mathrm{B}$, and $\mathrm{C}$ represent the dimensions of $\mathrm{CT}$ hyperdensity in three axes perpendicular to each other $[2,6]$.

\section{Statistical analysis}

SPSS version 22.0 (IBM SPSS Inc., Armonk, NY, USA) was used for statistical analyses. Categorical variables were assessed using Pearson's and Fisher's exact tests, and continuous variables were assessed using the Mann-Whitney $U$ test. A $p$-value of less than 0.05 was considered statistically significant.

\section{RESULTS}

\section{Comparison of clinical characteristics}

Overall, 38 patients with TICH in the frontal lobe were included in this study. The clinical characteristics of both groups are summarized in Table 1 . The mean age of the CUFC group was higher than that of the SOKA group (64.6 vs. 57.6 years, respectively). Of the patients, 28 (73.7\%) were men and $10(26.3 \%)$ were women, and the frequency of TBI in men was higher than that in women. The rate of concomitant extracranial injury was similar for CUFC group (23.3\%) and SOKA group (25\%). The difference in age, sex, and concomitant extracranial injury 

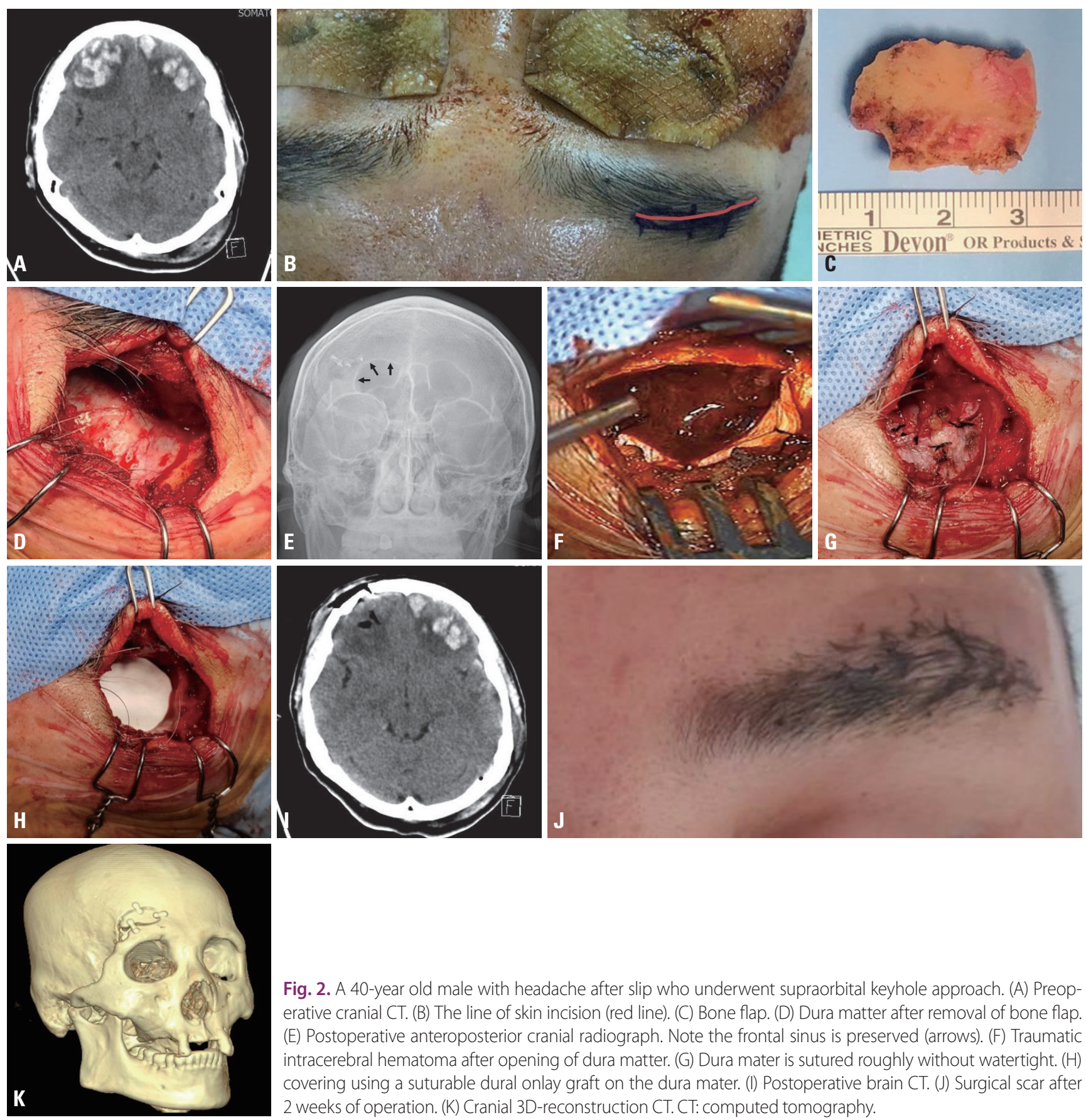

Fig. 2. A 40-year old male with headache after slip who underwent supraorbital keyhole approach. (A) Preoperative cranial CT. (B) The line of skin incision (red line). (C) Bone flap. (D) Dura matter after removal of bone flap. (E) Postoperative anteroposterior cranial radiograph. Note the frontal sinus is preserved (arrows). (F) Traumatic intracerebral hematoma after opening of dura matter. (G) Dura mater is sutured roughly without watertight. (H) covering using a suturable dural onlay graft on the dura mater. (I) Postoperative brain CT. (J) Surgical scar after 2 weeks of operation. (K) Cranial 3D-reconstruction CT. CT: computed tomography.

between the two groups were not statistically significant ( $p=0.265, p=1.000$, and $p=1.000)$. The most common cause of injury was a slip (50.0\%; $\mathrm{n}=19)$, followed by traffic accident $(34.2 \% ; \mathrm{n}=13)$, and falls $(15.8 \% ; \mathrm{n}=6)$. The initial GCS score of the CUFC group (9.8 \pm 3.3 years) was lower than that of the SOKA group (14.0 \pm 1.5 years).
Operative time and length of hospitalization of the CUFC group (214.1 \pm 80.4 minute and 31.6 \pm 26.7 days) was also shorter than that of the SOKA group $(76.6 \pm 6.4$ minute and $10.5 \pm 1.5$ days). The difference in the initial GCS score, operative time, and length of hospitalization between the two groups were statistically significant 
Table 1. Comparison of clinical characteristics between CUFC and SOKA group

\begin{tabular}{|c|c|c|c|}
\hline Characteristic & CUFC $(n=30)$ & SOKA $(n=8)$ & $p$-value \\
\hline Age (years) & $64.5 \pm 11.9$ & $57.6 \pm 19.5$ & 0.265 \\
\hline Sex & & & 1.000 \\
\hline Male:female & $22: 8$ & $6: 2$ & \\
\hline Concomitant extracranial injury & & & 1.000 \\
\hline Yes & 7 & 2 & \\
\hline No & 23 & 6 & \\
\hline Cause of injury & & & $<0.001$ \\
\hline Fall & 4 & 2 & \\
\hline Slip & 15 & 4 & \\
\hline Traffic accident & 11 & 2 & \\
\hline Initial GCS score & $9.8 \pm 3.3$ & $14.0 \pm 1.5$ & 0.002 \\
\hline Operative time (minutes) & $214.1 \pm 80.4$ & $76.6 \pm 6.4$ & $<0.001$ \\
\hline Length of hospitalization (days) & $31.6 \pm 26.7$ & $10.5 \pm 1.5$ & 0.005 \\
\hline Outcome & & & 0.164 \\
\hline Survival & 22 & 8 & \\
\hline Death & 8 & 0 & \\
\hline GCS score at discharge & $12.7 \pm 2.1$ & $14.3 \pm 1.2$ & 0.087 \\
\hline
\end{tabular}

CUFC: conventional unilateral frontal craniotomy, SOKA: supraorbital key-hole approach, GCS: Glasgow coma scale.

( $p=0.002, p<0.001$, and $p=0.005$ ). The mortality of CUFC group $(26.7 \%)$ was higher than that of the SOKA group $(0 \%)$, but this difference was not statistically significant $(p=0.164)$. The GCS score at discharge of the CUFC group (12.7 \pm 2.1$)$ was slightly lower than that of the SOKA group (14.3 \pm 1.2$)$, but this difference was not statistically significant $(p=0.087)$.

\section{Comparison of radiological features}

Associated skull fracture showed similar ratios in both CUFC (63.3\%) and SOKA (62.5\%) groups. Table 2 shows the comparison of radiological features of both groups. Specifically, the preoperative amount of TICH on CT markedly decreased postoperatively in both CUFC ( $46.0 \pm 30.7$ and $15.2 \pm 25.7$, respectively) and SOKA (43.6 \pm 9.9 and $8.5 \pm 4.5$, respectively) groups. The percentage of complete removal of hematoma for CUFC group $(40.0 \%)$ was quite higher than that for the SOKA group (12.5\%). The preoperative midline shifting of the hematoma on CT markedly decreased postoperatively in both CUFC (7.9 \pm 3.5 and $4.6 \pm 3.9$, respectively) and
SOKA (6.4 \pm 0.9 and $1.1 \pm 0.8$, retrospectively) groups. In the CUFC group, postoperative delayed hematoma in the surgical field was identified in six of 24 patients $(25 \%)$ and in one of eight patients in the SOKA group (12.5\%). All postoperative delayed hematomas were treated without surgical treatment. However, all radiological features between the two groups including associated skull fracture, amount of pre- and postoperative hematoma, percentage of complete hematoma removal, pre- and postoperative midline shifting of the hematoma, and development of postoperative delayed hematoma were not statistically significant ( $p=0.849, p=0.449 p=0.222$, and $p=1.000$ ).

\section{DISCUSSION}

TICH is defined as hematoma of a size greater than $2 \mathrm{~cm}$ in diameter that is not in contact with the brain surface and is found in $15 \%$ of autopsy cases of severe TBI $[1,6]$. TICHs are usually due to mechanisms such as gross hemorrhage caused by shear damage to small intraparenchy- 
Table 2. Comparison of radiological features between CUFC and SOKA groups

\begin{tabular}{|c|c|c|c|}
\hline Characteristic & CUFC $(n=30)$ & SOKA $(n=8)$ & $p$-value \\
\hline Associated skull fracture & & & 0.849 \\
\hline No & 11 & 3 & \\
\hline Unilateral & 17 & 4 & \\
\hline Bilateral & 2 & 1 & \\
\hline Amount of hematoma & & & 0.449 \\
\hline Preoperative & $46.0 \pm 30.7$ & $43.6 \pm 9.9$ & \\
\hline Postoperative & $15.2 \pm 25.7$ & $8.5 \pm 4.5$ & \\
\hline Complete removal of hematoma & & & 0.222 \\
\hline Yes & 12 & 1 & \\
\hline No & 18 & 7 & \\
\hline Midline shifting (mm) & & & 0.449 \\
\hline Preoperative & $7.9 \pm 3.5$ & $6.4 \pm 0.9$ & \\
\hline Postoperative & $4.6 \pm 3.9$ & $1.1 \pm 0.8$ & \\
\hline Postoperative delayed hematoma & & & 1.000 \\
\hline Yes & 6 & 1 & \\
\hline No & 24 & 7 & \\
\hline
\end{tabular}

CUFC: conventional unilateral frontal craniotomy, SOKA: supraorbital keyhole approach.

mal vessels or brain lacerations. Unlike cerebral contusions, TICH tends to appear homogeneously hyperdense on CT. TICH tends to have less edema around the lesion than with contusions and occur usually in the frontal or temporal lobes [6]. Zhang et al. [3] described that the surgical outcome of SOKA for evacuation of TICH in the frontal lobe was not significantly different from that of CUFC. In their paper, SOKA has a shorter operative time, less intraoperative blood loss, and shorter length of hospitalization than CUFC. Additionally, the incidence rate of postoperative bleeding, intracranial infection, and GCS scores after the 6-month follow-up between patients treated with SOKA or CUFC was not significantly different. Zhang et al. [3] insisted that the choice of SOKA should be based on the surgeon's experience and degree of bilateral frontal contusion because brain edema is the most common problem that can occur during TBI surgery.

With the development of microscope-assisted surgical techniques, some surgeons have performed evacuation of hematoma using small craniotomy under microscope [3]. These techniques have several benefits, including a shorter operative time, less tissue damage and blood loss, and faster postoperative recovery $[3,5,6]$. The SOKA is a minimally invasive surgery that can access mainly tumorous lesion of the anterior cranial fossa or aneurysms of the anterior circulation $[4,5]$. In the SOKA surgery, the operator did not suture the dura mater with watertight closure. Although the dura mater was sutured roughly and only covered with a suturable dural onlay graft on the dura mater, no CSF leakage was found in all SOKA cases. We were able to shorten the operative time using a gelantin-thrombin matrix sealant to facilitate hemostasis and suturable dural onlay graft. Although the learning curve and surgical technique for minimally invasive surgery is essential, the development of various surgical materials including these hemostatic agents and grafts has also made minimally invasive surgery easier, faster, and safer.

Moreover, we performed BHAD under local anesthesia in patients who refused surgery under general anesthesia because of the various reasons mentioned above (Fig. 3). Table 3 shows the clinical characteristics of patients who underwent BHAD. Most of the patients were elderly, hospitalized for traffic accidents, and taking medication 

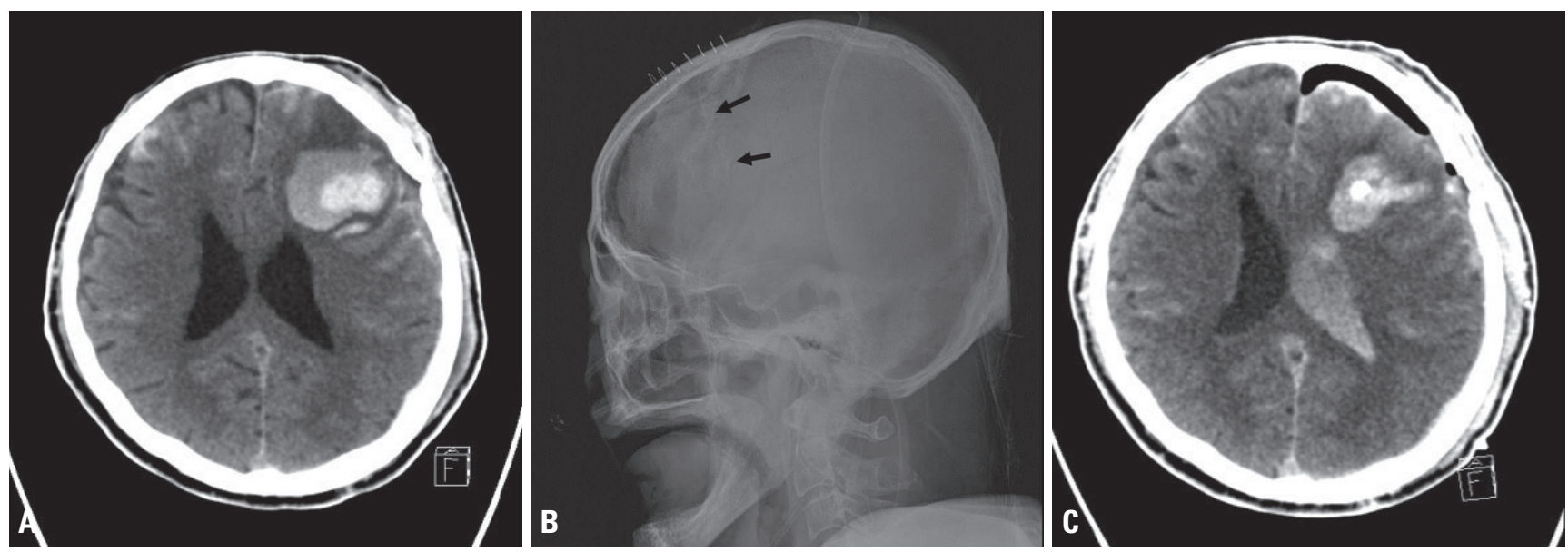

Fig. 3. A 82-year old female with stupor after traffic accident who underwent burr hole aspiration and drainage. (A) Preoperative cranial CT. (B) Postoperative lateral cranial X-ray shows the burr hole catheter (black arrows). (C) Postoperative brain CT. CT: computed tomography.

Table 3. Clinical characteristics of patients who underwent burr hole aspiration

\begin{tabular}{|lccccccccc|}
\hline No & Age & Sex & $\begin{array}{c}\text { Mental } \\
\text { status }\end{array}$ & GCS & Mechanism & $\begin{array}{c}\text { Concomitant extracranial injury } \\
\text { or associated disease }\end{array}$ & $\begin{array}{c}\text { Operation } \\
\text { time } \\
\text { (minutes) }\end{array}$ & $\begin{array}{c}\text { Length of } \\
\text { hospitalization (days) }\end{array}$ & Outcome \\
\hline 1 & 58 & M & Stupor & E2V(T)M5 & TA & Hemopneumothorax, femur fracture & 20 & 61 & Dull \\
\hline 2 & 83 & M & Drowsy & E3V4M6 & NA & CABG, Alzheimer's disease & 20 & 14 & Dull \\
\hline 3 & 96 & F & Drowsy & E3V3M6 & TA & Multiple contusions on whole body & 25 & 3 & Death \\
\hline 4 & 70 & M & Stupor & E2V2M5 & TA & CKD stage 5 & 25 & 1 & Death \\
\hline 5 & 77 & M & Stupor & E2V3M5 & TA & Pancytopenia, schizophrenia & 20 & 56 & Vegetative \\
\hline 6 & 82 & F & Stupor & E2V3M5 & TA & Femur shaft fracture & 25 & 60 & Dull \\
\hline 8 & 77 & M & Drowsy & E3V5M6 & Slip & Seizure, Parkinson's disease & 30 & 30 & Dull \\
\hline 9 & 75 & M & Drowsy & E3V5M6 & NA & Clavicle fracture & 25 & 10 & Death \\
\hline
\end{tabular}

CABG: coronary artery bypass graft, CKD: chronic kidney disease, F: female, NA: non-available, M: male; TA: traffic accident, GCS: Glasgow coma scale, E: eye, V: verbal, M: motor response.

for serious chronic diseases (such as coronary artery bypass graft surgery due to obstructive coronary disease, Alzheimer's disease, chronic kidney disease, pancytopenia, schizophrenia, seizure, and Parkinson's disease). The mean age was $77.3 \pm 10.9$ years, and the male ratio was $75 \%$. The mental status was drowsy in four patients and stupor in the other four patients. The initial GCS score was 11.4 \pm 2.1 , which was higher than that of CUFC

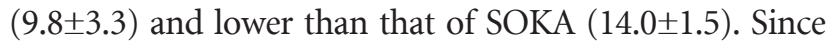
most of the patients were hospitalized due to traffic accidents, there were many accompanying extracranial injuries (hemopneumothorax, femur or clavicle fractures, multiple contusions on whole body, etc.). The operative time (23.8 \pm 3.5 minutes) was very short compared to that of CUFC or SOKA, and the preparation time was also short. The length of hospitalization was $29.4 \pm 26.1$ days, which varied according to the patient's condition. Three patients died, and mortality was $37.5 \%$, which was higher than that of CUFC (26.7\%), and one patient progressed to a vegetative state (GCS 4). Because BHAD was performed on patients who had poor preoperative conditions, selection bias would have had a significant impact on patients' outcomes. BHAD may be another surgical treatment option that can achieve some degree of decompression in a short time under various adverse conditions in which general anesthesia is difficult to perform. How- 
ever, this procedure should be used only in very limited cases because it is difficult to obtain satisfactory removal of hematoma or decompression for increased ICP, and the possibility of rebleeding also can be high.

Our experience of 46 patients with TICH in the frontal lobe with any of the three different surgical methods enabled us to obtain valuable findings. CUFC has the following advantages: 1) wide surgical field of view, 2) hematomas and hemostasis can be easily removed, 3) unexpected brain swelling or massive hemorrhage can be easily handled, and 4) the surgeon is familiar with this approach; hence, beginners can relatively perform the procedure easily. Meanwhile, SOKA has the following advantages: 1) short preparation and operative time; 2) minimal bleeding and tissue damage due to minimal invasion; 3) short hospitalization due to rapid recovery after surgery; and 4) no watertight closure of the dura mater is needed. The disadvantages of CUFC can be the advantages of SOKA, while the disadvantages of SOKA can be the advantages of CUFC. As mentioned earlier, in case of SOKA, the novice operator is not familiar with the technique compared to that in the CUFC; hence, the novice operator is likely to have difficulty in managing an unexpected intraoperative event, such as severe bleeding or brain swelling. However, the authors suggest that SOKA may be more advantageous than CUFC in terms of hematoma evacuation in carefully selected patients with brain CT showing the following findings: 1) TICH is well loculated; 2) TICH is located mainly in the frontal base, thereby allowing easy access for the surgical approach; 3 ) there is less contusion around TICH; and 4) there is less cerebral edema.

The advantages of BHAD should be noted as well. 1) The preparation and operative time are extremely short. 2) It can be performed under local anesthesia. 3) It can be performed immediately in an emergency when craniotomy is impossible. However, although there is no definite statistical, BHAD is expected to have higher morbidity and mortality than the other two procedures with CUFC and SOKA; therefore, more prospective studies will be needed in the future.

\section{Limitations}

Notably, this study has some limitations. First, this is a retrospective study, not a well-designed study, and has a large selection bias. Second, our investigation was based only on patients' medical record; thus, the inclusion criteria are unclear. In other words, this study did not provide clear criteria for surgical indication in patients with TICH in the frontal lobe. Third, the sample size was small, and long-term follow-up was not performed, and there was no comparative study of long-term outcomes, such as at 3 months or 6 months after surgery.

\section{CONCLUSION}

Despite the abovementioned limitations, the author compared and described the three approaches of CUFC, SOKA, and BHAD for TCIH localized to the frontal lobe. We believe that this study is noteworthy, providing useful data that can be used as reference in future research on the surgical approach for TICH in the frontal lobe. Although it is difficult to insist that one particular approach is more useful than the other, we are confident that SOKA will have more advantages over CUFC in carefully selected patients depending on the surgical experience of a neurosurgeon. Future studies should clarify the surgical indication, advantages, and disadvantages of each approach to TICH.

\section{REFERENCES}

1. McCormick WF. Pathology of closed head injury. In: Wilkins RH, Rengachary SS, eds. Neurosurgery. 2nd ed. New York: Mc-Graw-Hill;1996:2639-66.

2. Hung KS, Liang CL, Wang CH, Chang HW, Park N, Juo SH. Outcome after traumatic frontal intracerebral haemorrhage: a comparison of unilateral and bilateral haematomas. J Clin Neurosci 2004;11:849-53.

3. Zhang S, Qian C, Sun G, Li X. Clinical application of the supraorbital key-hole approach to the treatment of unilateral-dominant bilateral frontal contusions. Oncotarget 2017;8:48343-9.

4. Wiedemayer H, Sandalcioglu IE, Wiedemayer H, Stolke D. The supraorbital keyhole approach via an eyebrow incision for resection of tumors around the sella and the anterior skull base. Minim Invasive Neurosurg 2004;47:221-5. 
Eun Sung Park, et al. Surgical Approach for Frontal TICH

5. Mori K. Keyhole concept in cerebral aneurysm clipping and tumor removal by the supraciliary lateral supraorbital approach. Asian J Neurosurg 2014;9:14-20.
6. Le TH, Gean AD. Imaging of traumatic brain injury. In: Winn HR, ed. Youmans neurological surgery. 6th ed. Philadelphia: Elsevier/Saunders;2011:3342-61. 